\title{
Wide QRS-T angle and low T wave amplitude are associated with the presence of myocardial expansion as measured by extracellular volume fraction with cardiac MRI
}

\author{
Karolina M Zareba ${ }^{1 *}$, Akira Wada' ${ }^{1}$ Xiaojuan Xia², Jean-Philippe Couderc ${ }^{2}$, Subha V Raman ${ }^{1}$ \\ From 19th Annual SCMR Scientific Sessions \\ Los Angeles, CA, USA. 27-30 January 2016
}

\section{Background}

Extracellular matrix expansion as measured by extracellular volume fraction (ECV) with cardiac MRI (CMR) is a strong predictor of clinical outcomes. The aim of our study was to evaluate the relationship between EKG parameters and the presence of myocardial expansion as measured by ECV.

\section{Methods}

We studied 90 consecutive patients without myocardial infarctions (mean age 53 years, $50 \%$ female) referred for CMR with T1 mapping that had a 12 lead EKG within 90 days. Numerous EKG parameters were evaluated visually and electronically (heart rate, PR duration, QRS amplitude and duration, QRS morphology, QRS-T angle, QRS fragmentation, T wave amplitude, QTc duration, corrected $\mathrm{QT}$ peak duration, $\mathrm{T}$ peak $\mathrm{T}$ end) to determine their association with myocardial fibrosis as measured by ECV.

\section{Results}

There were 40 (44\%) patients with an elevated ECV $\geq 29 \%$. Patients with an elevated ECV were more likely to have a history of congestive heart failure ( $63 \%$ vs $16 \%, p=0.000)$, had lower ejection fraction ( $44 \%$ vs $56 \%, \mathrm{p}=0.000)$, and had more non-ischemic scar $(63 \%$ vs $24 \%, \mathrm{p}=0.000)$ as noted with late gadolinium enhancement (LGE). In patients with an elevated ECV, QRS Cornell amplitude (1.7 vs. $1.2 \mathrm{mV}, \mathrm{p}=0.018)$, and QRS-T angle $\left(112^{\circ}\right.$ vs. $57^{\circ}$, $\mathrm{p}=0.000)$ were significantly higher, while $\mathrm{T}$ wave amplitude ( 45 vs. $229 \mu \mathrm{V}, \mathrm{p}=0.002$ ) was significantly lower. Multivariate logistic regression revealed that QRS-T angle $\geq 100^{\circ}$ (OR 4.0, $\left.\mathrm{p}=0.015\right)$ and $\mathrm{T}$ wave amplitude $\leq$ $170 \mu \mathrm{V}(\mathrm{OR} 4.2, \mathrm{p}=0.011)$ were highly associated with an elevated ECV after adjustment for clinical covariates (heart failure, ejection fraction, LGE, bundle branch block).

\section{Conclusions}

Wide QRS-T angle and low $\mathrm{T}$ wave amplitude were independently associated with the presence of myocardial expansion as measured by ECV. Prospective evaluation of these parameters can aid in appropriate CMR referral for patients at risk of myocardial disease.

\section{Authors' details}

'Ohio State University, Columbus, OH, USA. ${ }^{2}$ University of Rochester, Rochester, NY, USA.

Published: 27 January 2016

\section{doi:10.1186/1532-429X-18-S1-P275}

Cite this article as: Zareba et al:: Wide QRS-T angle and low T wave amplitude are associated with the presence of myocardial expansion as measured by extracellular volume fraction with cardiac MRI. Journal of Cardiovascular Magnetic Resonance 2016 18(Suppl 1):P275.

Ohio State University, Columbus, OH, USA

Full list of author information is available at the end of the article 